\title{
Isolated aortic valve repair-how to do it and long-term results: external ring annuloplasty
}

\author{
Mustafa Zakkar ${ }^{1,2}$, Pouya Youssefi ${ }^{1}$, Isabelle Di Centa ${ }^{3}$, Nizar Khelil ${ }^{1}$, Mathieu Debauchez ${ }^{1}$, Emmanuel Lansac $^{1}$ \\ ${ }^{1}$ Department of Cardiac Surgery, L'Institut Mutualiste Montsouris, Paris, France; ${ }^{2}$ Department of Cardiovascular Sciences, University of Leicester, \\ Clinical Sciences Wing, Glenfield General Hospital, Leicester, UK; ${ }^{3}$ Vascular Surgery Unit, Hopital Foch, Suresnes, France \\ Correspondence to: Mr Mustafa Zakkar. Department of Cardiovascular Sciences, University of Leicester, Clinical Sciences Wing, Glenfield General \\ Hospital, Leicester, UK. Email: mustafazakkar@me.com.
}

Submitted Feb 07, 2019. Accepted for publication Mar 19, 2019.

doi: 10.21037/acs.2019.04.14

View this article at: http://dx.doi.org/10.21037/acs.2019.04.14

\section{Introduction}

The growing expertise in valve sparing root replacement over the years (1) has paved the way for developing isolated aortic valve (AV) leaflet repair as an alternative to replacement, especially when a standardized systematic approach in term of annulus and sinotubular junction (STJ) stabilization is utilized. Since 2003, we have developed a standardized systematic approach for AV repair which incorporates leaflet repair and the stabilization of annulus and STJ with double sub and supra (STJ) valvular external ring annuloplasties (Figure 1).

\section{Clinical vignette}

The first patient was a 61-year-old male who presented with worsening shortness of breath (NYHA IV). Preoperative transthoracic echocardiography (TTE) demonstrated a trileaflet AV (TAV) with severe aortic insufficiency (AI) (grade IV), left ventricular end-systolic diameter (LVESD) of $54 \mathrm{~mm}$, left ventricular end-diastolic diameter (LVEDD) of $70 \mathrm{~mm}$ and preserved LV function (50\%). Preoperative gated computed tomography (CT) showed normal sinus of Valsalva size $(36 \mathrm{~mm})$, STJ size of $30 \mathrm{~mm}$ and ascending aorta size of $31 \mathrm{~mm}$.

The second patient was a 54-year-old male with similar presentation. Preoperative TTE demonstrated a bicuspid AV (BAV) with severe AI (grade IV), LVESD of $50 \mathrm{~mm}$, LVEDD of $65 \mathrm{~mm}$ and preserved LV function (54\%). Preoperative gated CT showed normal sinus of Valsalva size $(34 \mathrm{~mm})$, STJ size of $29 \mathrm{~mm}$ and ascending aorta size of $30 \mathrm{~mm}$.

Both patients were discussed in a multidisciplinary meeting and deemed suitable for AV repair. The options of intervention were discussed in detail with the patient who opted for valve repair or replacement with bioprosthetic valve if the valve was not repairable on the table.

\section{Surgical technique}

\section{Preparation}

Pre-operative standard evaluation of patients includes a TTE and a gated CT scan. A detailed on-table transoesophageal echo (ToE) allows for a comprehensive assessment of the type of valve, the presence of leaflet prolapse, retraction, fenestrations and calcification and confirmation of the size of the annulus, root, STJ and ascending aorta diameter.

\section{Exposition}

Once the cross clamp is applied, a right superior pulmonary vent is placed and the aorta is open transversely $1 \mathrm{~cm}$ distal to STJ then cardioplegia is administered directly to both ostia. We routinely use Custodiol as a single dose cardioplegia which is usually sufficient for the whole procedure.

\section{Operation}

The valve systemically assessed to check the leaflets quality, free cusp margins and the presence of calcifications, fenestrations or retraction (cusp height of $16 \mathrm{~mm}$ or less in TAVs and $19 \mathrm{~mm}$ or less on the non-fused cusp for bicuspid 
valve) (2). The native aortic annulus diameter is measured with Hegar dilators and the size of the annuloplasty rings used is based on the size of the Hegar dilator (Table 1) aiming to downsize the ring by approximately one size ( $2 \mathrm{~mm})$ (compared to the Hegar measurement) in order to perform an annuloplasty that will reduce the native annulus diameter and increase the surface of coaptation.

For the placement of subvalvular U-anchoring stitches for ring annuloplasty, we use five threads of 2.0 coated polyester fibre pledgeted which are placed from the inside out as "U" stitches (width of $3 \mathrm{~mm}$ ) circumferentially in the subvalvular plane, clock-wise beginning from the non-coronary sinus. Three sutures are positioned $2 \mathrm{~mm}$ below the nadir of each cusp and two are placed at the base of the inter leaflet triangles between the non and left coronary sinuses and between the left and right coronary sinuses. A sixth suture is passed externally at the level of the inter leaflet triangle between the right and non-coronary sinus, without pinching inside in order to limit the risk of

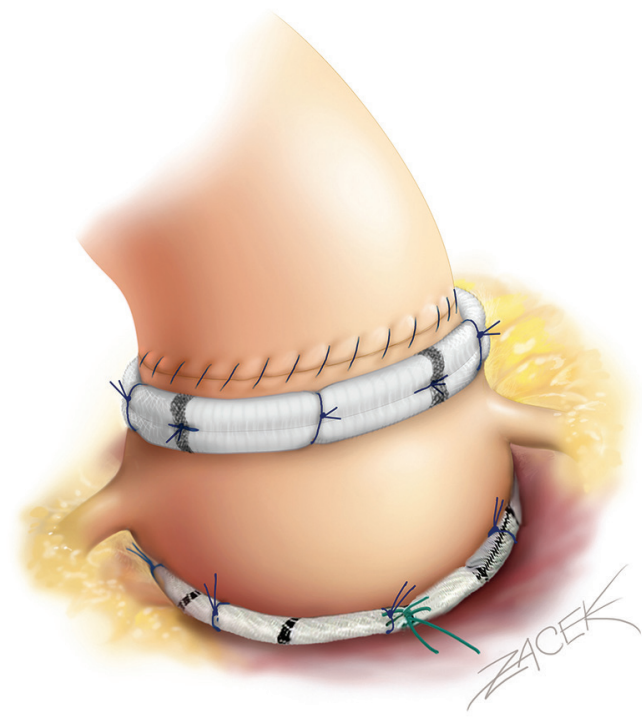

Figure 1 Double sub and supra valvular ring annuloplasty for isolated AI repair (drawing with kind permission of Dr Pavel Zacek). membranous septum or the bundle of His injury.

\section{Isolated TAV repair}

A polypropylene $6 / 0$ stay suture is passed through each noduli of Arantius. A grasper pulls outwards on the corresponding commissure while the two stitches at the level of the noduli of Arantia are retracted in the opposite direction. The excess length of free edge is then determined. The same step is then repeated for each hemicusp. Distance between the two stitches determines the area for the central plicating stitches to equalize each hemi-cusp free edge length (polypropylene 5 or $6 / 0$ ).

STJ ring placement is performed using six threads of 2.0 coated polyester fibre pledgeted which are placed from the inside out as " $U$ " stitches (width of $3 \mathrm{~mm}$ ) circumferentially. Commissures are placed symmetrically on the STJ ring at 120 degrees.

Commissural traction sutures are then placed to mimic a pressurized aortic root in order to measure the effective height of each cusp. A dedicated cusp caliper is used to evaluate any residual or induced cusp prolapse (Fehling Instruments, Karlstein, Germany) (2). Plicating stitches are added on the free edge of the culprit leaflet until an effective height of $9 \mathrm{~mm}$ is obtained.

\section{Isolated BAV repair}

The first step of valve repair consists of aligning the cusp free edge lengths along the cusps of reference which is the non fused one. A polypropylene 5 or $6 / 0$ stay suture is passed through each noduli of Arantius. A grasper pulls outwards on the corresponding commissure while the two stitches at the level of the noduli of Arantia are retracted in the opposite direction. The excess length of free edge is then determined. Any excess free margin length of the nonfused cusp is corrected through central plicating stitches (polypropylene 5 or $6 / 0$ ) or cusp resection.

In case of asymmetric commissural orientation $<170^{\circ}$, a plication of the sinus at raphe level is added in order to improve the symmetrical design of the repair. Plication

Table 1 Criteria for choice of the subvalvular aortic ring and sinotubular ring size

\begin{tabular}{lllll}
\hline \multirow{2}{*}{ Variables } & \multicolumn{3}{l}{ Surgical aortic annular size (Hegar) $(\mathrm{mm})$} & \\
\cline { 2 - 5 } & $25-27$ & $28-31$ & $32-35$ & $>35$ \\
\hline Sinotubular junction extra-aortic ring $(\mathrm{mm})$ & 25 & 27 & 29 & 31 \\
Subvalvular extra-aortic ring $(\mathrm{mm})$ & 25 & 27 & 29 & 31 \\
\hline
\end{tabular}


is performed from the external side with a couple of " $U$ " stitches of 2.0 coated polyester fibre pledgeted sutures from either side of the raphe. First stich is placed above the valve and the second above towards the STJ.

STJ ring placement is performed using six threads of 2.0 coated polyester fibre pledgeted which are placed from the inside out as " $U$ " stitches (width of $3 \mathrm{~mm}$ ) circumferentially. Commissures are placed symmetrically at $180^{\circ}$ for the two functional commissures on the STJ ring using the expandable external aortic ring (ExtraAortic, CORONEO, Inc.). Other stiches are displayed on the circumference taking particular care to avoid any compression of the coronary ostia.

Commissural traction stitches are placed and adequately pulled on the commissure at $180^{\circ}$ to mimic a pressurized aortic root. The measurement of the effective height is performed only on the non-coronary cusp with a dedicated caliper (Fehling Instruments, Karlstein, Germany). Plicating stitches are added on the free edge of the culprit leaflet only if the effective height is significantly lower than $9 \mathrm{~mm}$ in order to preserve as much as possible the length of the nonfused cusp for the long term opening of the valve.

Management of valve defect is performed using either glutaraldehyde treated autologous pericardial patch or with decellularized matrix patch such as CardioCel (Admedus Ltd). While waiting for an open version of the Extra aortic ring, the six anchoring " $U$ " stitches are passed around a Dacron ring $3 \mathrm{~mm}$ wide slice of a tube graft which is passed behind the right and left coronary arteries using right-angle blunt forceps. Ring is closed at the non-coronary sinus level. " $U$ " stitches are tied around the ring to secure it in subvalvular position.

\section{Completion}

The aortotomy is closed using polypropylene 4/0 running suture, the heart de-aired and cross-clamp removed. Postprocedural $\mathrm{ToE}$ is done before weaning off $\mathrm{CPB}$ to assess valve function, the size of the annulus and STJ and the presence and pathology of any degree of AI.

We do not accept any residual $\mathrm{AI}>\mathrm{II}$ and based on the ToE findings, we either re-clamp in case of eccentric jet due to residual prolapse and re-repair again (or replace) or we downsize the subvalvular ring by placing extra sutures at the non-coronary sinus level in case of central jet with residual annular dilation. A final ToE check is carried out once the patient is weaned off. We routinely obtain a pre-discharge TTE on all patients and again at 3 months postoperatively. Postoperative anticoagulation consisted of aspirin ( $75 \mathrm{mg} /$ day) administered for 3 months unless persistent atrial fibrillation had been present, in which case oral anticoagulation was used.

For further description of techniques and surgical management of specific valvular lesion sets, please see http://www.aorticvalverepair.net/.

\section{Comments}

\section{Clinical results}

Based on our experience with remodelling technique using an external ring annuloplasty, we opted to carry out a standardized approach for isolated AV repair supported by sub and supra valvular external ring annuloplasties (double ring). We stopped briefly using the STJ ring hoping to simplify the technique. We previously reported that isolated AV repair was associated with $97.5 \%$ overall survival at 7 years with no difference between the bicuspid and tricuspid valve. Freedom from AI $\geq$ grade 3 at 7 years was $82.2 \%$ and that the use of additional sinotubular junction ring (double sub- and supravalvular annuloplasty) tended to reduce recurrent $\mathrm{AI}$ when compared with single subvalvular annuloplasty (3). More recently we looked at the impact of STJ stabilization on the long-term durability from our series (93 patients out of a whole series of 482 patients) extracted from the AVIATOR registry. Our analysis suggests the long-term survival after AV repair is excellent and similar to sex and age matched population. Furthermore; our analysis suggests that the use of double ring annuloplasty was associated with $100 \%$ freedom from the recurrence of $\mathrm{AI} \geq$ grade 3 compared to $67 \%$ in the single annuloplasty group at 6 years $(\mathrm{P}=0.008)$. Moreover; the isolated use of double annuloplasty was correlated with $97 \%$ freedom from $\mathrm{AV}$ related re-intervention compared to $73 \%$ in the single annuloplasty group $(\mathrm{P}=0.02)$ at 6 years. Thus, we returned back to using double ring as a routine practice.

\section{Advantages}

The importance of dealing with two of the major components of the AV (annulus, and STJ) by using sub and supra commissural plicating stiches for AV repair has been established many years ago (4). The simple use of subcommissural plicating stiches has since been clearly demonstrated to be risk factor of reoperation and recurrent AI due re-dilatation of the aortic annulus (5-7). These 
serious problems resulted in some groups advocating valve sparing root replacement using the reimplantation technique in the presence of significant AI even when the root and ascending aorta are normal in size. Although this was associated with significant improvement of outcomes which can be due to the fact that the reimplantation technique not only achieve a subvalvular annuloplasty through the proximal suture but also a supravalvular stabilisation at STJ level thus restoring the ratio between STJ and annulus, however, it is difficult to justify or apply as a routine practice for non-dilated root.

\section{Caveats}

Experience with this technique remains limited and very long-term results will have to be studied as planned within the international AVIATOR registry from the Heart Valve Society (http://heartvalvesociety.org/AVIATOR/).

\section{Acknowledgments}

None.

\section{Footnote}

Conflicts of Interest: $\mathrm{E}$ Lansac has consultant agreements with CORONEO, Inc. (www.coroneo.com), in connection with the development of an aortic ring bearing the trade name "Extra-Aortic". The other authors have no conflicts of interest to declare.

\section{References}

1. Lansac E, Di Centa I, Sleilaty G, et al. Remodeling root repair with an external aortic ring annuloplasty. J Thorac Cardiovasc Surg 2017;153:1033-42.

2. Schäfers HJ, Bierbach B, Aicher D. A new approach to the assessment of aortic cusp geometry. J Thorac Cardiovasc Surg 2006;132:436-8.

3. Lansac E, Di Centa I, Sleilaty G, et al. Long-term results of external aortic ring annuloplasty for aortic valve repair. Eur J Cardiothorac Surg 2016;50:350-60.

4. Cabrol C, Cabrol A, Guiraudon G, et al. Treatment of aortic insufficiency by means of aortic annuloplasty. Arch Mal Coeur Vaiss 1966;59:1305-12.

5. de Kerchove L, Boodhwani M, Glineur D, et al. Valve sparing-root replacement with the reimplantation technique to increase the durability of bicuspid aortic valve repair. J Thorac Cardiovasc Surg 2011;142:1430-8.

6. Navarra E, El Khoury G, Glineur D, et al. Effect of annulus dimension and annuloplasty on bicuspid aortic valve repair. Eur J Cardiothorac Surg 2013;44:316-22; discussion 322-3.

7. de Kerchove L, Mastrobuoni S, Boodhwani M, et al. The role of annular dimension and annuloplasty in tricuspid aortic valve repair. Eur J Cardiothorac Surg 2016;49:42837; discussion 437-8.
Cite this article as: Zakkar M, Youssefi P, Di Centa I, Khelil $\mathrm{N}$, Debauchez M, Lansac E. Isolated aortic valve repairhow to do it and long-term results: external ring annuloplasty. Ann Cardiothorac Surg 2019;8(3):418-421. doi: 10.21037/ acs.2019.04.14 